\title{
New Criteria for Functions to Be in a Class of $p$-Valent Alpha Convex Functions
}

\author{
Muhammad Arif, ${ }^{1}$ Muhammad Ayaz, ${ }^{1}$ and Mohamed Kamal Aouf ${ }^{2}$ \\ ${ }^{1}$ Department of Mathematics, Abdul Wali Khan University, Mardan 23200, Pakistan \\ ${ }^{2}$ Faculty of Science, Mansoura University, Mansoura 35516, Egypt
}

Correspondence should be addressed to Muhammad Arif; marifmaths@yahoo.com

Received 5 August 2013; Accepted 30 August 2013

Academic Editors: M. Imdad and M.-S. Liu

Copyright (C) 2013 Muhammad Arif et al. This is an open access article distributed under the Creative Commons Attribution License, which permits unrestricted use, distribution, and reproduction in any medium, provided the original work is properly cited.

We obtain certain simple sufficiency criteria for a class of $p$-valent alpha convex functions. Many known results appear as special consequences of our work. Some applications of our work to the generalized integral operator are also given.

\section{Introduction}

Let $A_{p}(n)$ denote the class of functions $f(z)$ of the form

$$
f(z)=z^{p}+\sum_{k=p+n}^{\infty} a_{k} z^{k} \quad(p \in \mathbb{N}=\{1,2, \ldots\}),
$$

which are analytic and multivalent in the open unit disk $\mathbb{U}=$ $\{z \in \mathbb{C}:|z|<1\}$. We note that $A_{1}(n)=A(n)$ and $A_{1}(1)=A$. Also let $S_{p}^{*}(n, \beta), C_{p}(n, \beta)$ and $M_{p}(\alpha, n, \beta),(0 \leq \alpha \leq 1$, $0 \leq \beta<p, n, p \in \mathbb{N})$, denote the subclasses of $A_{p}(n)$ consisting of all functions $f(z)$, of the form (1), which are defined, respectively, by

$$
\begin{gathered}
\operatorname{Re} \frac{z f^{\prime}(z)}{f(z)}>\beta, \quad(z \in \mathbb{U}), \\
1+\operatorname{Re} \frac{z f^{\prime \prime}(z)}{f^{\prime}(z)}>\beta, \quad(z \in \mathbb{U}), \\
\operatorname{Re}\left\{(1-\alpha) \frac{z f^{\prime}(z)}{f(z)}+\alpha \frac{z f^{\prime \prime}(z)}{f^{\prime}(z)}\right\}>\beta-\alpha, \quad(z \in \mathbb{U}) .
\end{gathered}
$$

For $p=1$ and $n=1$, the above three classes reduce to the well-known classes of starlike, convex, and alpha convex functions of order $\beta$, respectively. We also note that $M_{1}(0, n, 0) \equiv S^{*}(n, 0), M_{1}(1, n, 0) \equiv C(n, 0)$.

Sufficient condition was studied by various authors for different subclasses of analytic and multivalent functions; for some of the related work, see [1-9]. The object of the present paper is to obtain sufficient conditions for the class $M_{p}(\alpha, n, \beta)$ of multivalent alpha convex functions of order $\beta$. We also consider some special cases of our results which lead to various interesting corollaries, and relevances of some of these with other known results are also mentioned.

We will assume throughout our discussion, unless otherwise stated, that $n \in \mathbb{N}, 0 \leq \alpha \leq 1,0 \leq \beta<p$, and $p \in \mathbb{N}$.

To obtain our main results, we need the following lemmas due to Mocanu [10].

Lemma 1. If $q(z) \in A(n)$ satisfies the condition

$$
\left|q^{\prime}(z)-1\right|<\frac{n+1}{\sqrt{(n+1)^{2}+1}}(z \in \mathbb{U}),
$$

then

$$
q(z) \in S^{*}(n, 0) .
$$

Lemma 2. If $q(z) \in A(n)$ satisfies the condition

$$
\left|\arg q^{\prime}(z)\right|<\frac{\pi}{2} \delta_{n} \quad(z \in \mathbb{U}),
$$

where $\delta_{n}$ is the unique root of the equation

$$
2 \tan ^{-1}\left[n\left(1-\delta_{n}\right)\right]+\pi\left(1-2 \delta_{n}\right)=0,
$$

then

$$
q(z) \in S^{*}(n, 0) .
$$




\section{Sufficient Conditions for}

the Class $M_{p}(\alpha, n, \beta)$

Theorem 3. If $f(z) \in A_{p}(n)$ satisfies

$$
\begin{aligned}
& \mid\left(\frac{f(z)}{z^{p}}\left(\frac{z f^{\prime}(z)}{p f(z)}\right)^{\alpha}\right)^{1 /(p-\beta)} \\
& \quad \times\left\{(1-\alpha) \frac{z f^{\prime}(z)}{f(z)}+\alpha \frac{z f^{\prime \prime}(z)}{f^{\prime}(z)}+\alpha-\beta\right\}-p+\beta \mid \\
& <\frac{n+1}{\sqrt{(n+1)^{2}+1}}(p-\beta) \quad(z \in \mathbb{U}),
\end{aligned}
$$

then $f(z) \in M_{p}(\alpha, n, \beta)$.

Proof. Let us set a function $q(z)$ by

$$
\begin{aligned}
q(z) & =z\left(\frac{f(z)}{z^{p}}\left(\frac{z f^{\prime}(z)}{p f(z)}\right)^{\alpha}\right)^{1 /(p-\beta)} \\
& =z+\frac{\alpha(p+n) a_{p+n}}{p(p-\beta)} z^{n+1}+\cdots,
\end{aligned}
$$

for $f(z) \in A_{p}(n)$. Then clearly (9) shows that $q(z) \in A(n)$. Differentiating (9) logarithmically, we have

$q^{\prime}(z)$

$$
=q(z)\left[\frac{1}{z}+\frac{1}{p-\beta}\left\{(1-\alpha) \frac{f^{\prime}(z)}{f(z)}+\alpha \frac{f^{\prime \prime}(z)}{f^{\prime}(z)}-\frac{p-\alpha}{z}\right\}\right],
$$

which gives

$$
\begin{aligned}
&\left|q^{\prime}(z)-1\right|=\mid\left(\frac{f(z)}{z^{p}}\left(\frac{z f^{\prime}(z)}{p f(z)}\right)^{\alpha}\right)^{1 /(p-\beta)} \frac{1}{p-\beta} \\
& \times\left\{(1-\alpha) \frac{z f^{\prime}(z)}{f(z)}+\alpha \frac{z f^{\prime \prime}(z)}{f^{\prime}(z)}+\alpha-\beta\right\}-1 \mid .
\end{aligned}
$$

Thus using (8), we have

$$
\left|q^{\prime}(z)-1\right| \leq \frac{n+1}{\sqrt{(n+1)^{2}+1}}, \quad(z \in \mathbb{U}) .
$$

Hence, using Lemma 1 , we have $q(z) \in S^{*}(n, 0)$.

From (10), we can write

$$
\frac{z q^{\prime}(z)}{q(z)}=1+\frac{1}{p-\beta}\left\{(1-\alpha) \frac{z f^{\prime}(z)}{f(z)}+\alpha \frac{z f^{\prime \prime}(z)}{f^{\prime}(z)}+\alpha-p\right\} .
$$

Since $q(z) \in S^{*}(n, 0)$, it implies that $\operatorname{Re}\left(z q^{\prime}(z) / q(z)\right)>0$. Therefore, we get

$$
\begin{aligned}
& \frac{1}{p-\beta} \operatorname{Re}\left\{(1-\alpha) \frac{z f^{\prime}(z)}{f(z)}+\alpha\left(1+\frac{z f^{\prime \prime}(z)}{f^{\prime}(z)}\right)-\beta\right\} \\
& =\operatorname{Re} \frac{z q^{\prime}(z)}{q(z)}>0,
\end{aligned}
$$

or

$$
\frac{1}{p-\beta} \operatorname{Re}\left\{(1-\alpha) \frac{z f^{\prime}(z)}{f(z)}+\alpha\left(1+\frac{z f^{\prime \prime}(z)}{f^{\prime}(z)}\right)-\beta\right\}>0,
$$

and this implies that $f(z) \in M_{p}(\alpha, n, \beta)$.

By taking $\alpha=0$ and $\alpha=1$ in Theorem 3, we obtain Corollaries 4 and 5 proved by Goyal et al. [6].

Corollary 4. If $f(z) \in A_{p}(n)$ satisfies

$$
\begin{aligned}
& \left|\left(\frac{f(z)}{z}\right)^{1 /(p-\beta)}\left\{z^{(1-\beta) /(p-\beta)} \frac{f^{\prime}(z)}{f(z)}-\beta z^{(1-p) /(p-\beta)}\right\}-p+\beta\right| \\
& \quad<\frac{n+1}{\sqrt{(n+1)^{2}+1}}(p-\beta) \quad(z \in \mathbb{U}),
\end{aligned}
$$

for $0 \leq \beta<p$, then $f(z) \in S_{p}^{*}(n, \beta)$.

Corollary 5. If $f(z) \in A_{p}(n)$ satisfies

$$
\begin{aligned}
& \left|\left\{\frac{\left(f^{\prime}(z)\right)^{\beta+1-p}}{p z^{p-1}}\right\}^{1 /(p-\beta)}\left\{z f^{\prime \prime}(z)+(1-\beta) f^{\prime}(z)\right\}-p+\beta\right| \\
& <\frac{n+1}{\sqrt{(n+1)^{2}+1}}(p-\beta) \quad(z \in \mathbb{U}),
\end{aligned}
$$

for $0 \leq \beta<p$, then $f(z) \in C_{p}(n, \beta)$.

Further if we take $n=1$ and $p=1$ in Corollaries 4 and 5, we get the following result proved by Uyank et al. [9] .

Corollary 6. If $f(z) \in A$ satisfies

$$
\begin{aligned}
& \left|\left(\frac{f(z)}{z}\right)^{1 /(1-\beta)}\left\{\frac{z f^{\prime}(z)}{f(z)}-\beta\right\}-1+\beta\right| \\
& <\frac{2}{\sqrt{5}}(1-\beta) \quad(z \in \mathbb{U}),
\end{aligned}
$$

for $0 \leq \beta<1$, then $f(z) \in S^{*}(\beta)$.

Corollary 7. If $f(z) \in A$ satisfies

$$
\begin{aligned}
& \left|\left(f^{\prime}(z)\right)^{\beta /(1-\beta)}\left\{f^{\prime}(z)+\frac{1}{1-\beta} z f^{\prime \prime}(z)\right\}-1\right| \\
& <\frac{2}{\sqrt{5}} \quad(z \in \mathbb{U}),
\end{aligned}
$$

for $0 \leq \beta<1$, then $f(z) \in C(\beta)$. 
Remark 8. If we put $\beta=0$ and $p=1$ in Corollaries 4 and 5, we get the result proved by Mocanu [7] and Nunokawa et al. [8], respectively.

Theorem 9. If $f(z) \in A_{p}(n)$ satisfies

$$
\begin{aligned}
& \mid \arg \left\{\frac{f(z)}{z^{p}}\left(\frac{z f^{\prime}(z)}{p f(z)}\right)^{\alpha}\right\} \\
& +(p-\beta) \arg \left\{(1-\alpha) \frac{z f^{\prime}(z)}{f(z)}+\alpha \frac{z f^{\prime \prime}(z)}{f^{\prime}(z)}+\alpha-\beta\right\} \mid \\
& \quad<\frac{\pi}{2} \delta_{n}(p-\beta) \quad(z \in \mathbb{U}),
\end{aligned}
$$

where $\delta_{n}$ is the unique root of $(6)$, then $f(z) \in M_{p}(\alpha, n, \beta)$.

Proof. Let us set

$$
\begin{aligned}
q(z) & =z\left(\frac{f(z)}{z^{p}}\left(\frac{z f^{\prime}(z)}{p f(z)}\right)^{\alpha}\right)^{1 /(p-\beta)} \\
& =z+\frac{\alpha(p+n) a_{p+n}}{p(p-\beta)} z^{n+1}+\cdots,
\end{aligned}
$$

for $f(z) \in A_{p}(n)$. Then clearly (9) shows that $q(z) \in A(n)$.

Differentiating (9), we have

$q^{\prime}(z)$

$$
\begin{aligned}
=\frac{q(z)}{z}\{ & \frac{1}{(p-\beta)} \\
& \left.\times\left\{(1-\alpha) \frac{z f^{\prime}(z)}{f(z)}+\alpha \frac{z f^{\prime \prime}(z)}{f^{\prime}(z)}+\alpha-p\right\}+1\right\},
\end{aligned}
$$

which gives

$$
\begin{aligned}
& \left|\arg q^{\prime}(z)\right| \\
& =\mid \arg \frac{q(z)}{z} \\
& \quad+\arg \left\{(1-\alpha) \frac{z f^{\prime}(z)}{f(z)}+\alpha \frac{z f^{\prime \prime}(z)}{f^{\prime}(z)}+\alpha-\beta\right\} \mid .
\end{aligned}
$$

Thus using (8), we have

$$
\left|\arg q^{\prime}(z)\right| \leq \frac{\pi}{2} \delta_{n} \quad(z \in \mathbb{U}),
$$

where $\delta_{n}$ is the root of (6). Hence, using Lemma 2, we have $q(z) \in S^{*}(n, 0)$. Now by the same arguments as in the proof of Theorem 3, we obtain the required result.

Making $\alpha=0$ in Theorem 9, we have the following.
Corollary 10. If $f(z) \in A_{p}(n)$ satisfies

$$
\begin{aligned}
& \left|\arg \left(\frac{f(z)}{z^{p}}\right)+(p-\beta) \arg \left\{\frac{z f^{\prime}(z)}{f(z)}-\beta\right\}\right| \\
& \quad<\frac{\pi}{2} \delta_{n}(p-\beta) \quad(z \in \mathbb{U}),
\end{aligned}
$$

then $f(z) \in S_{p}^{*}(n, \beta)$.

Further if we take $n=1=p$ in Corollary 10, we get the following result proved by Uyank et al. [9].

Corollary 11. If $f(z) \in A$ satisfies

$$
\begin{aligned}
& \left|\arg \left(\frac{f(z)}{z}\right)+(1-\beta) \arg \left\{\frac{z f^{\prime}(z)}{f(z)}-\beta\right\}\right| \\
& \quad<\frac{\pi}{2} \delta_{1}(1-\beta) \quad(z \in \mathbb{U}),
\end{aligned}
$$

where $\delta_{1}$ is the unique root of the equation

$$
2 \tan ^{-1}\left[\left(1-\delta_{1}\right)\right]+\pi\left(1-2 \delta_{1}\right)=0,
$$

then $f(z)$ belongs to the class of starlike functions of order $\beta$.

Taking $\alpha=1$ in Theorem 9, we have the following.

Corollary 12. If $f(z) \in A_{p}(n)$ satisfies

$$
\begin{aligned}
& \left|\arg \left(\frac{f^{\prime}(z)}{p z^{p-1}}\right)+(p-\beta) \arg \left\{\frac{z f^{\prime \prime}(z)}{f^{\prime}(z)}+1-\beta\right\}\right| \\
& \quad<\frac{\pi}{2} \delta_{n}(p-\beta), \quad(z \in \mathbb{U}),
\end{aligned}
$$

then $f(z) \in C_{p}(n, \beta)$.

Remark 13. If we take $n=1$ and $p=1$ in Corollary 12, we get the result proved in [9], and further for $\beta=0$, we get the result proved by Mocanu [10].

\section{Generalized Integral Operator}

For $f(z) \in A_{p}(n)$, we consider

$$
G(z)=\left\{\delta \int_{0}^{z} t^{\delta-1}\left(\frac{f(t)}{t^{p}}\right)^{\gamma} d t\right\}^{1 / \delta}=z+\frac{\gamma a_{p+n}}{(n+\delta)} z^{n+1}+\cdots
$$

Clearly $G(z) \in A(n)$, and when $p=1, \gamma=1$, and $\delta=1$, then (29) reduces to the well-known Alexander integral operator [11].

Theorem 14. If $\gamma \geq \delta / p, \delta>0$, and $f(z) \in A_{p}(n)$ satisfy

$$
\left|\left(\frac{f(z)}{z^{p}}\right)^{\gamma / \delta}\left\{1+\frac{\gamma}{\delta}\left(\frac{z f^{\prime}(z)}{f(z)}-p\right)\right\}-1\right| \leq \frac{(n+1)}{\sqrt{(n+1)^{2}+1}},
$$

then $f(z) \in S_{p}^{*}(n, 0)$. 
Proof. From (29), we get

$$
G^{\delta-1}(z) G^{\prime}(z)=z^{\delta-1}\left(\frac{f(z)}{z^{p}}\right)^{\gamma}
$$

Differentiating (31), logarithmically, we get

$$
(\delta-1) \frac{G^{\prime}(z)}{G(z)}+\frac{G^{\prime \prime}(z)}{G^{\prime}(z)}=(\delta-1) \frac{1}{z}+\gamma\left(\frac{f^{\prime}(z)}{f(z)}-\frac{p}{z}\right) .
$$

Then by simple computation, we have

$$
\begin{aligned}
& \mid \frac{G(z)}{z}\left(\frac{z G^{\prime}(z)}{G(z)}\right)^{1 / \delta} \\
& \left.\quad \times\left\{\left(1-\frac{1}{\delta}\right) \frac{z G^{\prime}(z)}{G(z)}+\frac{1}{\delta}\left(\frac{z G^{\prime \prime}(z)}{G^{\prime}(z)}+1\right)\right\}-1 \mid\right\} \mid \\
& \quad=\left|\left(\frac{f(z)}{z^{p}}\right)^{\gamma / \delta}\left\{1+\frac{\gamma}{\delta}\left(\frac{z f^{\prime}(z)}{f(z)}-p\right)\right\}-1\right| \\
& \quad \leq \frac{(n+1)}{\sqrt{(n+1)^{2}+1}},
\end{aligned}
$$

where we have used (30). By using Theorem 3 with $p=1$, $\beta=0$, and $\alpha=1 / \delta$, we have $G(z) \in M_{1}(1 / \delta, n, 0)$.

From (32), we can write

$$
\begin{aligned}
& \operatorname{Re}\left\{\left(1-\frac{1}{\delta}\right) \frac{z G^{\prime}(z)}{G(z)}+\frac{1}{\delta}\left(\frac{z G^{\prime \prime}(z)}{G^{\prime}(z)}+1\right)\right\} \\
& =\frac{\gamma}{\delta} \operatorname{Re} \frac{z f^{\prime}(z)}{f(z)}-\frac{p \gamma}{\delta}+1,
\end{aligned}
$$

or

$$
\operatorname{Re} \frac{z f^{\prime}(z)}{f(z)}>\left(p-\frac{\delta}{\gamma}\right) \quad\left(\text { since } G(z) \in M_{1}\left(\frac{1}{\delta}, n, 0\right)\right)
$$

which shows that $f(z) \in S_{p}^{*}(n, 0)$, where $\gamma \geq \delta / p$.

Theorem 15. If $\gamma \geq \delta / p, \delta>0$, and $f(z) \in A_{p}(n)$ satisfy

$$
\left|\arg \left(\frac{f(z)}{z^{p}}\right)^{\gamma / \delta}+\arg \left\{\frac{\gamma}{\delta}\left(\frac{z f^{\prime}(z)}{f(z)}-p\right)+1\right\}\right|<\frac{\pi}{2} \delta_{n},
$$

where $\delta_{n}$ is the unique root of $(6)$, then $f(z) \in S_{p}^{*}(n, 0)$.

Proof. The result follows on similar lines as in the last theorem using Theorem 9 with $p=1, \beta=0$, and $\alpha=$ $1 / \delta$.

\section{Conflict of Interests}

The authors have no conflict of interests.

\section{References}

[1] H. Al-Amiri and P. T. Mocanu, "Some simple criteria of starlikeness and convexity for meromorphic functions," Mathematica, vol. 37, no. 60, pp. 11-21, 1995.

[2] M. Arif, "Sufficiency criteria for a class of p-valent analytic functions of complex order," Abstract and Applied Analysis, vol. 2013, Article ID 517296, 4 pages, 2013.

[3] M. Arif, I. Ahmad, M. Raza, and K. Khan, "Sufficient condition of a subclass of analytic functions defined by Hadamard product," Life Science Journal, vol. 9, no. 4, pp. 2487-2489, 2012.

[4] M. Arif, M. Raza, S. Islam, J. Iqbal, and F. Faizullah, "Some sufficient conditions for spirallike functions with argument properties," Life Science Journal, vol. 9, no. 4, pp. 3770-3773, 2012.

[5] B. A. Frasin, "Some sufficient conditions for certain integral operators," Journal of Mathematical Inequalities, vol. 2, no. 4, pp. 527-535, 2008.

[6] S. P. Goyal, S. K. Bansal, and P. Goswami, "Extensions of sufficient conditions for starlikeness and convexity of order $\alpha$ for multivalent function," Applied Mathematics Letters, vol. 25, no. 11, pp. 1993-1998, 2012.

[7] P. T. Mocanu, "Some starlikeness conditions for analytic functions," Revue Roumaine de Mathématique Pures et Appliquées, vol. 33, pp. 117-124, 1988.

[8] M. Nunokawa, S. Owa, Y. Polattoglu, M. Caglar, and E. Y. Duman, "Some sufficient conditions for starlikeness and convexity," Turkish Journal of Mathematics, vol. 34, pp. 333-337, 2010.

[9] N. Uyank, M. Aydogan, and S. Owa, "Extensions of sufficient conditions for starlikeness and convexity of order $\alpha$," Applied Mathematics Letters, vol. 24, no. 8, pp. 1393-1399, 2011.

[10] P. T. Mocanu, "Some simple criteria for starlikeness and convexity," Libertas Mathematica, vol. 13, pp. 27-40, 1993.

[11] J. W. Alexander, "Function which map the interior of the unit circle upon simple regions," Annals of Mathematics, vol. 17, no. 1, pp. 12-22, 1915. 


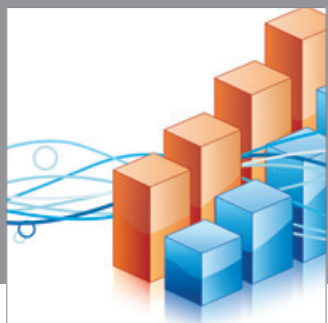

Advances in

Operations Research

mansans

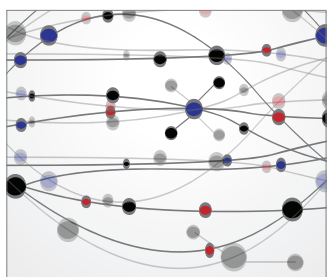

The Scientific World Journal
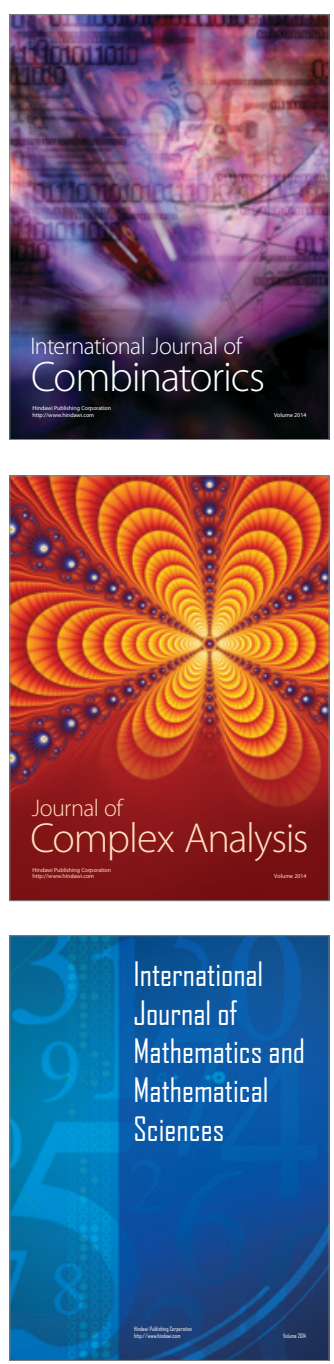
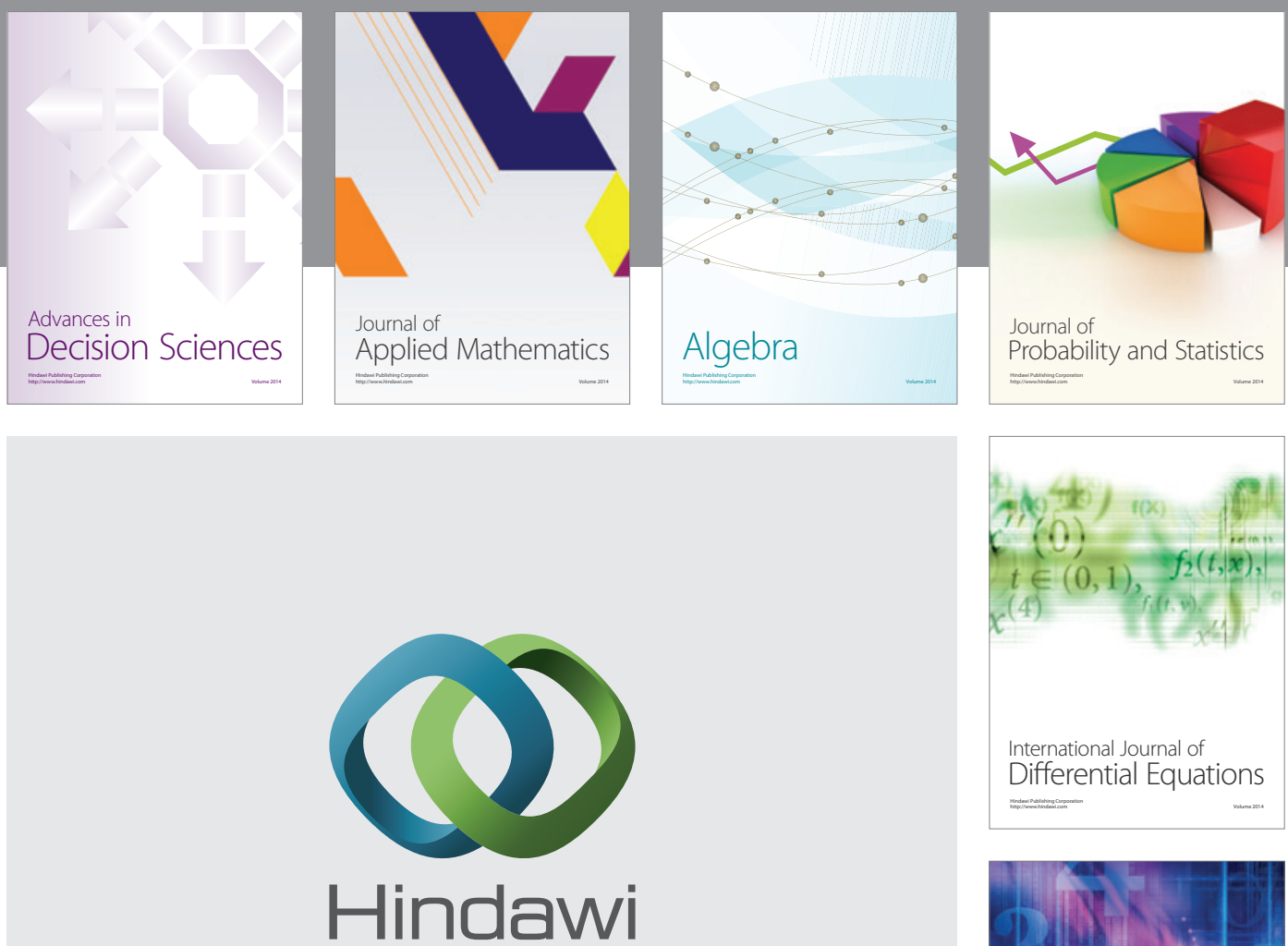

Submit your manuscripts at http://www.hindawi.com
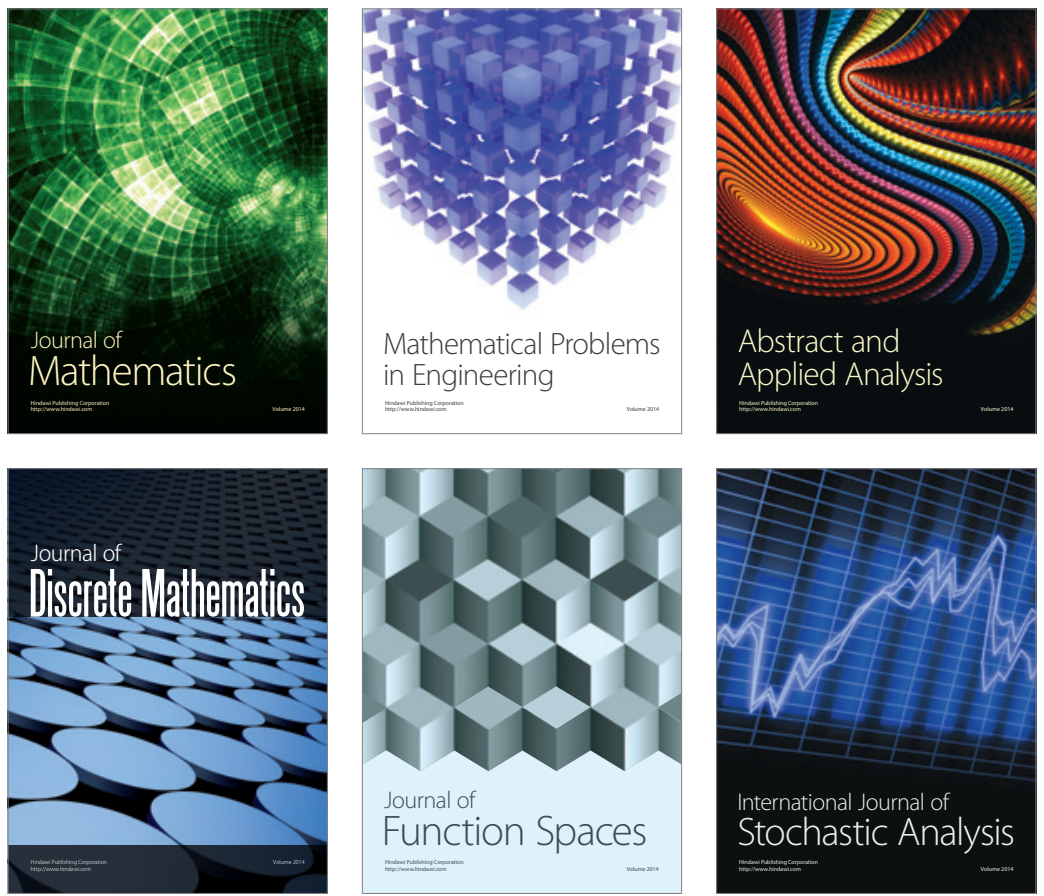

Journal of

Function Spaces

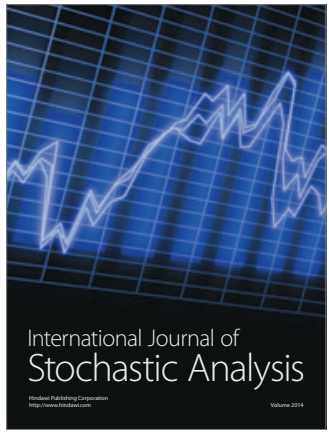

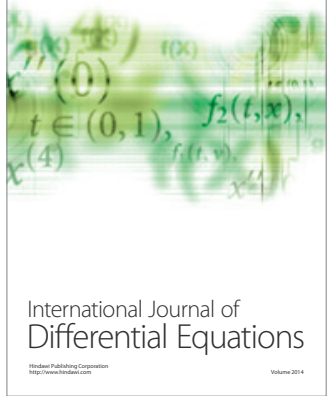
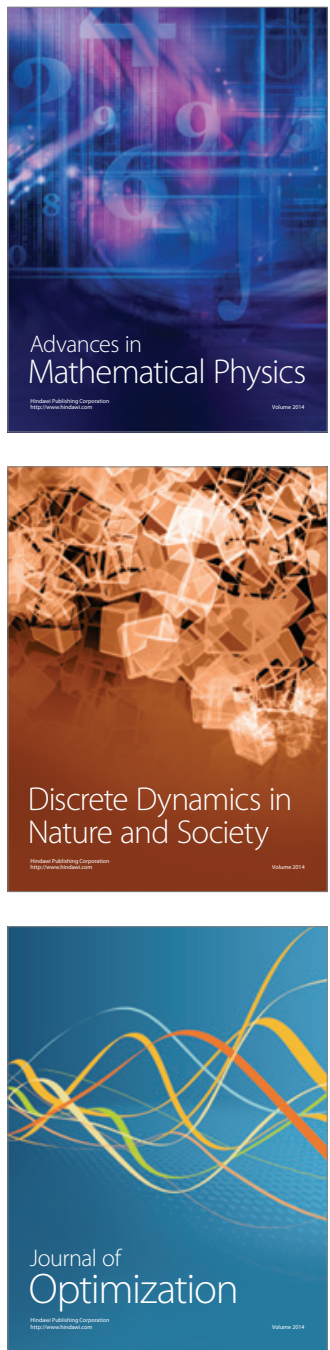